\title{
Time-Dependent Simulations of Fast-Wave Heated High-Non- Inductive-Fraction H-Mode Plasmas in the National Spherical Torus Experiment Upgrade
}

\author{
Gary Taylor ${ }^{1, *}$, Nicola Bertelli ${ }^{1}$, Stefan P. Gerhardt ${ }^{1}$, Joel C. Hosea ${ }^{1}$, Dennis Mueller ${ }^{1}$, Rory J. Perkins ${ }^{1}$, \\ Francesca M. Poli ${ }^{1}$, James R. Wilson ${ }^{1}$, and Roger Raman $^{2}$ \\ ${ }^{1}$ Princeton Plasma Physics Laboratory, Princeton University, Princeton, New Jersey 08543, USA \\ ${ }^{2}$ William E. Boeing Department of Aeronautics and Astronautics, University of Washington, Seattle, Washington 98195, USA
}

\begin{abstract}
MHz}$ fast-wave heating may be an effective tool for non-inductively ramping low-current plasmas to a level suitable for initiating up to $12 \mathrm{MW}$ of neutral beam injection on the National Spherical Tokamak Experiment Upgrade (NSTX-U). Previously on NSTX $30 \mathrm{MHz}$ fast wave heating was shown to efficiently and rapidly heat electrons; at the NSTX maximum axial toroidal magnetic field $\left(B_{T}(0)\right)$ of $0.55 \mathrm{~T}, 1.4 \mathrm{MW}$ of $30 \mathrm{MHz}$ heating increased the central electron temperature from 0.2 to $2 \mathrm{keV}$ in $30 \mathrm{~ms}$ and generated an H-mode plasma with a non-inductive fraction $\left(f_{N I}\right) \sim 0.7$ at a plasma current $\left(I_{p}\right)$ of $300 \mathrm{kA}$. NSTX-U will operate at $B_{T}(0)$ up to $1 \mathrm{~T}$, with up to $4 \mathrm{MW}$ of $30 \mathrm{MHz}$ power $\left(P_{r f}\right)$. Predictive TRANSP free boundary transport simulations, using the TORIC full wave spectral code to calculate the fast-wave heating and current drive, have been run for NSTX-U $I_{p}=300 \mathrm{kA} \mathrm{H}$-mode plasmas. Favorable scaling of $f_{N I}$ with $30 \mathrm{MHz}$ heating power is predicted, with $f_{N I} \geq 1$ for $P_{r f} \geq 2 \mathrm{MW}$.
\end{abstract}

\section{Introduction}

A Fusion Nuclear Science Facility based on the spherical tokamak concept needs to operate with little or no inductive drive from a central solenoid [1]. A long-term research goal of the National Spherical Tokamak Experiment Upgrade (NSTX-U) [2] is to develop fully non-inductive plasmas, and the NSTX-U fast-wave heating system may be an effective tool for noninductively ramping low-current plasmas to a level suitable for initiating up to $12 \mathrm{MW}$ of neutral beam injection [3]. On NSTX, $30 \mathrm{MHz}$ fast-wave heating efficiently and rapidly heated electrons; at the NSTX maximum axial toroidal field $\left(B_{T}(0)\right)$ of $0.55 \mathrm{~T}, 1.4 \mathrm{MW}$ of fast-wave heating increased the central electron temperature $\left(T_{e}(0)\right)$ from 0.2 to $2 \mathrm{keV}$ in $30 \mathrm{~ms}$ and generated an H-mode plasma (NSTX shot \#138506) with a non-inductive fraction $\left(f_{N I}\right) \sim 0.7$ at a plasma current $\left(I_{p}\right)=300 \mathrm{kA}$ [4]. The NSTX-U fast-wave heating system will couple up to $4 \mathrm{MW}$ of RF power $\left(P_{r f}\right)$ and will operate again at $30 \mathrm{MHz}$, which is the $4^{\text {th }}$ harmonic of deuterium at the maximum NSTX-U $B_{T}(0)$ of $1 \mathrm{~T}$. Predictive TRANSP free boundary transport simulations [5] have been run for NSTX-U $I_{p}=300 \mathrm{kA} \mathrm{H}$-mode plasmas in order to study the dependence of $f_{N I}$ on $B_{T}(0)$ and $P_{r f}$. The TRANSP simulations used the TORIC full wave spectral code [6] to calculate the fast-wave heating and current drive. The transport in the simulations was benchmarked to the experimental results obtained on NSTX. This paper presents the results of these simulations and the implications for using $30 \mathrm{MHz}$ power on NSTX-U to ramp $I_{p}$ non-inductively.

\section{Predictive modelling assumptions}

The thermal transport model used in the predictive TRANSP simulations was the Multimode MMM7.1 model [7]. This model gave reasonably good agreement to plasma parameters (eg. $f_{N I}, T_{e}(0)$ and plasma pressure) obtained experimentally during NSTX shot \#138506 for a NSTX-U simulation with similar $B_{T}(0)$ and $P_{r f}$. Effective charge, impurity, rotation and other profiles were taken largely from NSTX plasma shot \#142305, a plasma that was part of an experimental campaign to support NSTX-U and next-step Spherical Tokamak devices [8].

All the simulations used a $k_{/ /}=8 \mathrm{~m}^{-1}$ antenna phasing, the current drive antenna phasing used for shot \#138506. The RF pulse was ramped up to the flat top level in $30 \mathrm{~ms}$, starting at $100 \mathrm{~ms}$. This RF power ramp rate was typical for experiments on NSTX.

In the simulations the electron density profile and central density $\left(n_{e}(0)\right)$ were initially chosen to be similar to shot \#138506, which had $n_{e}(0)=1.15 \times 10^{19} \mathrm{~m}^{-3}$. The density in the simulation was ramped to this level in $100 \mathrm{~ms}$, at that time a H-mode transition was imposed by flattening the density profile, as shown in figure 1 . However when $B_{T}(0)>0.75 \mathrm{~T}$ it was found that when using this relatively low density the plasma became unstable and the simulations terminated prematurely,

\footnotetext{
Corresponding author: gtaylor@pppl.gov
} 
typically only $\sim 100 \mathrm{~ms}$ after the start of the RF pulse. Increasing $n_{e}(0)$ to $1.43 \times 10^{19} \mathrm{~m}^{-3}$ resulted in stable plasma simulations up to $P_{r f}=4 \mathrm{MW}$ and $B_{T}(0)=1 \mathrm{~T}$. Simulations were run for $P_{r f}=1.4,2,3$ and $4 \mathrm{MW}$, and for plasmas having $B_{T}(0)=0.5,0.65,0.75,0.89$ and $1 \mathrm{~T}$.

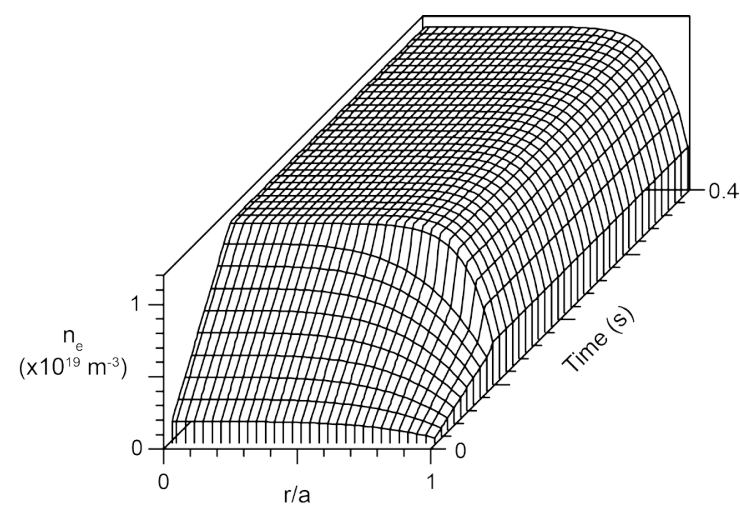

Fig. 1. Time evolution of the density profile used in the $\mathrm{n}_{\mathrm{e}}(0)=1.15 \times 10^{19} \mathrm{~m}^{-3}$ TRANSP simulation.

\section{Results}

Figure 2 summarizes the predictive TRANSP simulation results for a NSTX-U plasma with the same

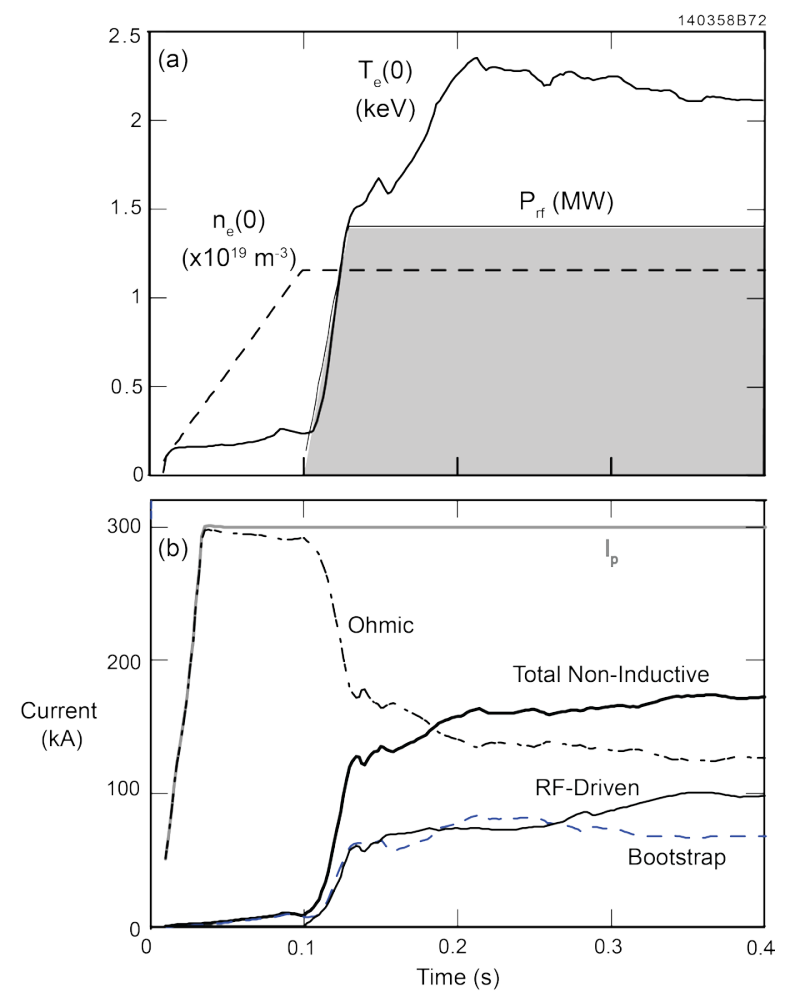

Fig. 2. Predictive TRANSP results for an $I_{p}=300 \mathrm{kA}$, $B_{T}(0)=0.5 \mathrm{~T}$ NSTX-U plasma with $P_{r f}=1.4 \mathrm{MW}$. (a) Time evolution of $n_{e}(0), T_{e}(0)$ and $P_{r f}$. (b) Time evolution of various currents predicted by TRANSP. The predicted $T_{e}(0)$ and $f_{N I}$ are similar to NSTX shot \#138506.
$P_{r f}, \quad I_{p}$ and $n_{e}(0)$ as NSTX shot \#138506, and $B_{T}(0)=0.5 \mathrm{~T}$, compared to $B_{T}(0)=0.55 \mathrm{~T}$ for shot \#138506. As shown in figure 2(a), $T_{e}(0)$ during the simulation was 2.2-2.4 keV, compared to $2.5-3 \mathrm{keV}$ during shot\# 138506. $f_{N I}$ during the simulated NSTX-U plasma reached 0.6, compared to a fluctuating $f_{N I}=0.7 \pm 0.2$ during shot \#138506. One notable difference was that the bootstrap current was larger than the current driven directly by RF power in shot \#138506. The fluctuation in $f_{N I}$ during shot \#138506 is due entirely to large fluctuations in the plasma pressure gradient resulting in fairly rapid changes in bootstrap current. In contrast, in the NSTX-U plasma simulation shown in figure 2(b) the bootstrap current is comparable to the RF-driven current and neither non-inductive currents have significant fluctuations.

The best value of $f_{N I}$ reached during the lower density, $n_{e}(0)=1.15 \times 10^{19} \mathrm{~m}^{-3}$ simulations was 1.7 . This high value of $f_{N I}$ was achieved for a simulation with
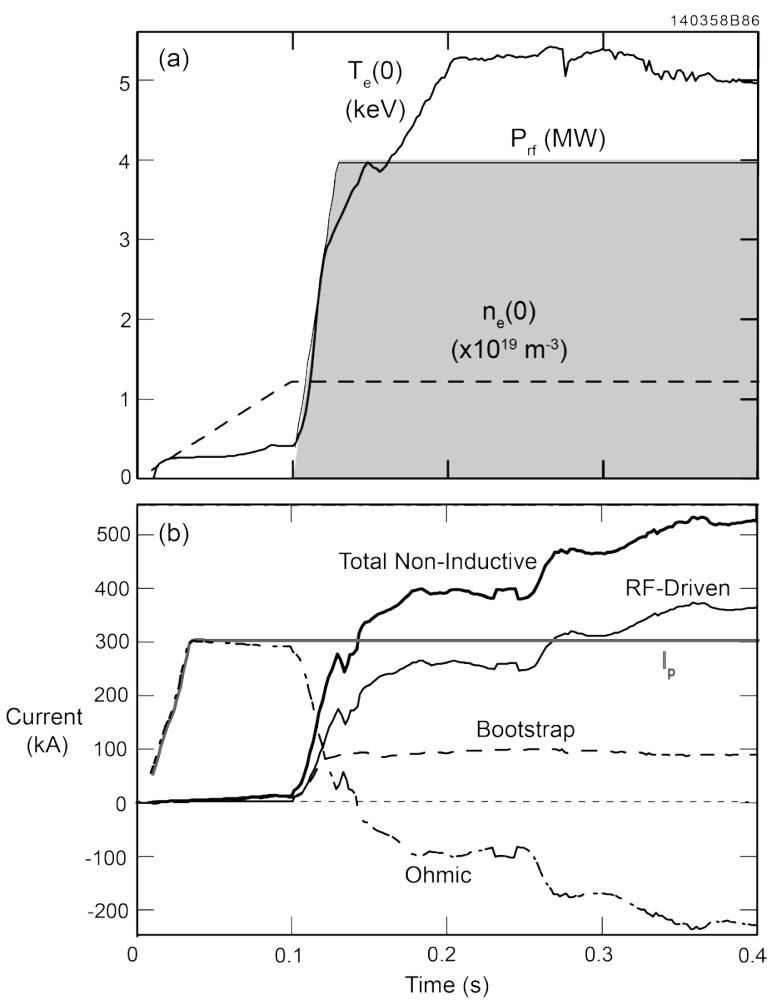

Fig. 3. Predictive TRANSP results for an $I_{p}=300 \mathrm{kA}$, $B_{T}(0)=0.65 \mathrm{~T}$ NSTX-U plasma with $P_{r f}=4 \mathrm{MW}$. (a) Time evolution of $n_{e}(0), T_{e}(0)$ and $P_{r f}$. (b) Time evolution of various currents predicted by TRANSP.

$B_{T}(0)=0.65 \mathrm{~T}$ and $P_{r f}=4 \mathrm{MW}$. The results from this simulation are summarized in figures 3 and 4 . Figure 3(a) shows the time evolution of $T_{e}(0)$ reaches $5 \mathrm{keV}$. As shown in figure $3(\mathrm{~b})$, the bootstrap current remains constant around $100 \mathrm{kA}$ and the RF-driven current reaches $370 \mathrm{kA}$ at $0.4 \mathrm{~s}$. Figure 4(a) shows $T_{e}$ at the start of the RF pulse (dashed line) and at $0.4 \mathrm{~s}$ (solid line) for the case shown in figure 3 . The $T_{e}$ profile becomes very peaked during the RF pulse (figure 4(a)). Figure 4(b) 
shows the area integrated current densities at $0.4 \mathrm{s.} 90 \%$ of the RF-driven current is within $r / a=0.2$, while $90 \%$ of the bootstrap current is generated within $r / a=0.5$. Because the RF heating was strongly peaked near the axis this results in the development of a highly peaked $T_{e}(R)$ profile (figure 4(a)) which further increases the RF-driven current near the magnetic axis. This peaking

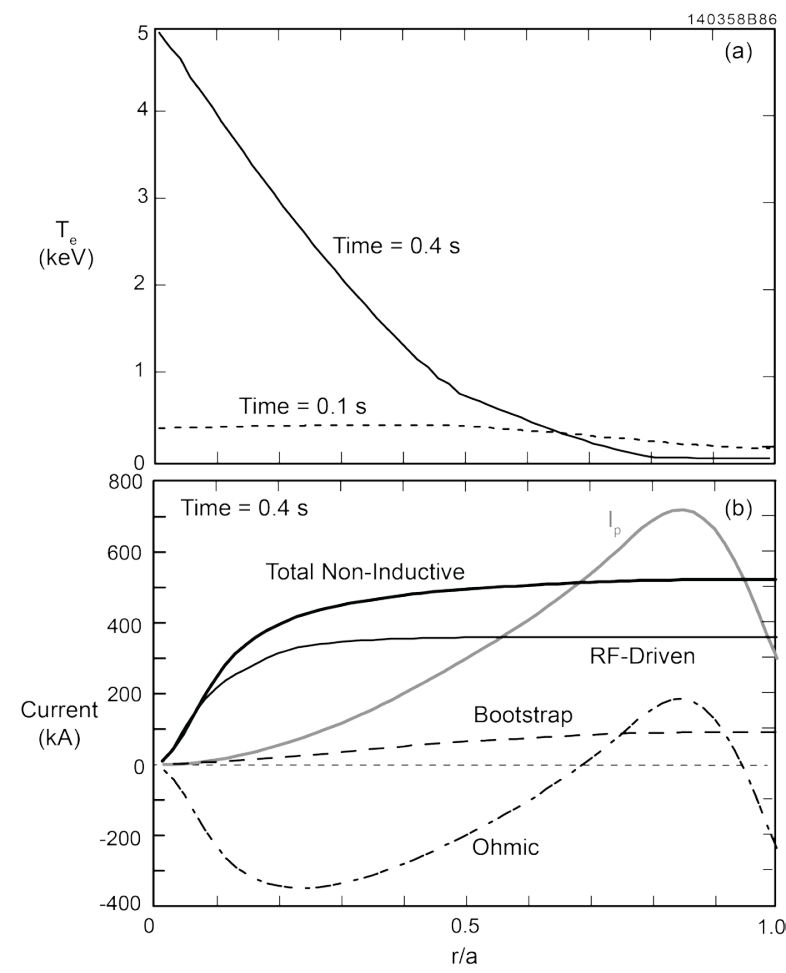

Fig. 4. (a) Electron temperature profile at $0.1 \mathrm{~s}$, the start of the RF pulse (dashed line) and at $0.4 \mathrm{~s}$ (solid line). (b) Area integrated currents versus normalized minor radius for the case shown in figure 3 at 0.4 s. $90 \%$ of the RFdriven current is located inside $r / a=0.3$.

of $T_{e}(0)$ and the RF-driven current at higher $\mathrm{P}_{\mathrm{rf}}$, particularly at higher $B_{T}(0)$, caused the plasma simulation to become unstable in the first $100 \mathrm{~ms}$ of the $\mathrm{RF}$ pulse, prematurely terminating the simulation, as mentioned earlier. This behaviour was also seen in NSTX shot \#138506. Although it didn't cause the plasma to disrupt it did result in a magneto-hydrodynamic reconnection in the plasma core that rapidly reduced the stored energy resulting in the plasma moving radially and briefly tripping the RF heating pulse.

All the lower density, $n_{e}(0)=1.15 \times 10^{19} \mathrm{~m}^{-3}$ simulations that didn't terminate by $\sim 0.2 \mathrm{~s}$ reached a quasi-steady state condition by $0.4 \mathrm{~s}$. Figure 5 shows the dependence of $f_{N I}$ at $0.4 \mathrm{~s}$ on $P_{r f}$ for the $n_{e}(0)=1.15 \times 10^{19} \mathrm{~m}^{-3}$ simulations. Also plotted in this figure is the $f_{N I}$ achieved during NSTX shot \#138506. At $P_{r f}=1.4 \mathrm{MW}$ all the simulations have an $f_{N I} \sim 0.6$, independent of $B_{T}(0)$, and in fairly good agreement with the $f_{N I}=0.7 \pm 0.2$ achieved experimentally during NSTX shot \#138506. Increasing $P_{r f}$ from 1.4 to $4 \mathrm{MW}$ for the $B_{T}(0)=0.5 \mathrm{~T}$ simulation approximately doubled $f_{N I}$. For the $B_{T}(0)=0.65 \mathrm{~T}$ and $0.75 \mathrm{~T}$ simulations $f_{N I}$ increased by 2.8 and 2.4 , respectively. The $B_{T}(0)=0.89 \mathrm{~T}$ simulation became unstable at $\sim 0.2 \mathrm{~s}$ when $P_{r f}$ was

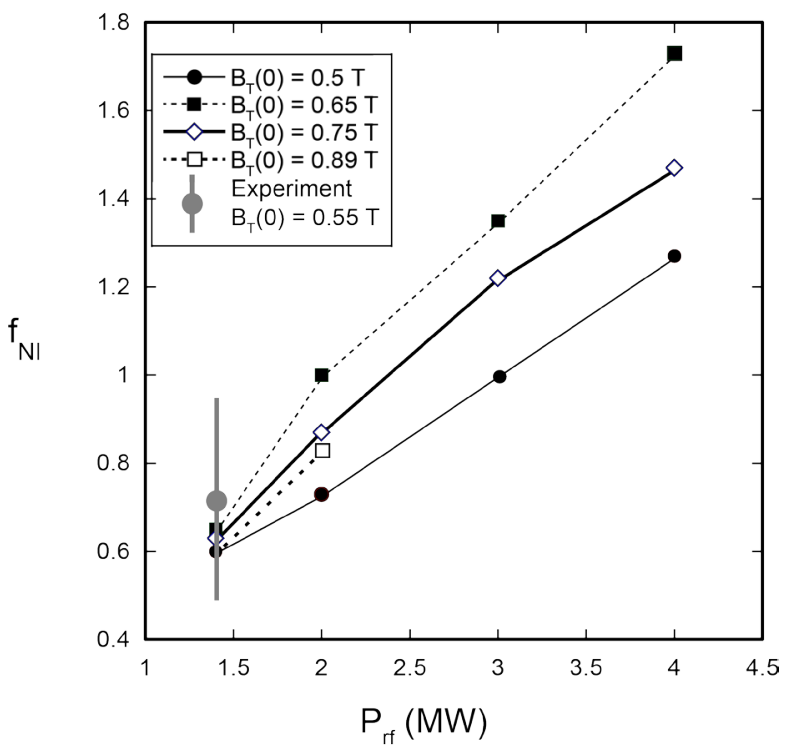

Fig. 5. $f_{N I}$ at $0.4 \mathrm{~s}$ versus $P_{r f}$ for plasma simulations with $n_{e}(0)=1.15 \times 10^{19} \mathrm{~m}^{-3}$. Also plotted in this figure is the $f_{N I}$ achieved during NSTX shot \#138506 (grey filled circle with range during the shot indicated by the vertical bar).

increased above $2 \mathrm{MW}$ and the $B_{T}(0)=1 \mathrm{~T}$ simulations were all unstable early in the RF pulse.

Figure 6 shows the dependence of $f_{N I}$ at $0.4 \mathrm{~s}$ on $B_{T}(0)$ for the $n_{e}(0)=1.15 \times 10^{19} \mathrm{~m}^{-3}$ simulations. Increasing $B_{T}(0)$ from 0.5 to $0.65 \mathrm{~T}$ is predicted to significantly increase $f_{N I}$, particularly at the higher $P_{r f}$. However, $f_{N I}$

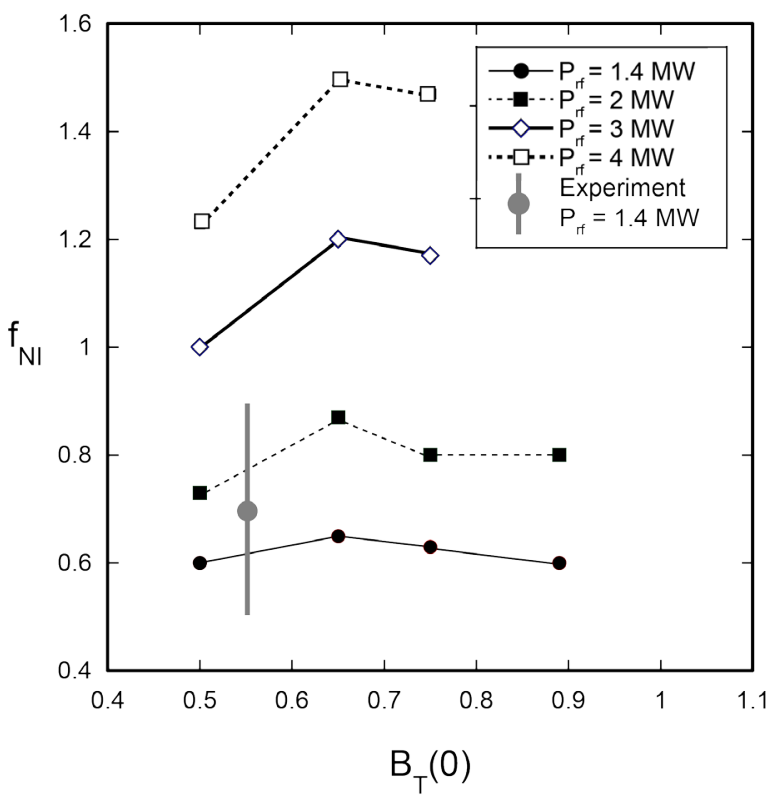

Fig. 6. $f_{N I}$ versus $\mathrm{B}_{\mathrm{T}}(0)$ for the $n_{e}(0)=1.15 \times 10^{19} \mathrm{~m}^{-3}$ plasma simulations. Also plotted in this figure is the $f_{N I}$ achieved during NSTX shot\# 138506 (grey filled circle with range during the shot indicated by the vertical bar). 
decreases above $B_{T}(0)=0.65 \mathrm{~T}$ at all values of $P_{r f}$.

For the $n_{e}(0)=1.15 \times 10^{19} \mathrm{~m}^{-3}$ simulations fully noninductive operation is predicted with as little as $2 \mathrm{MW}$ of RF power for a $B_{T}(0)=0.65 \mathrm{~T}$ plasma,

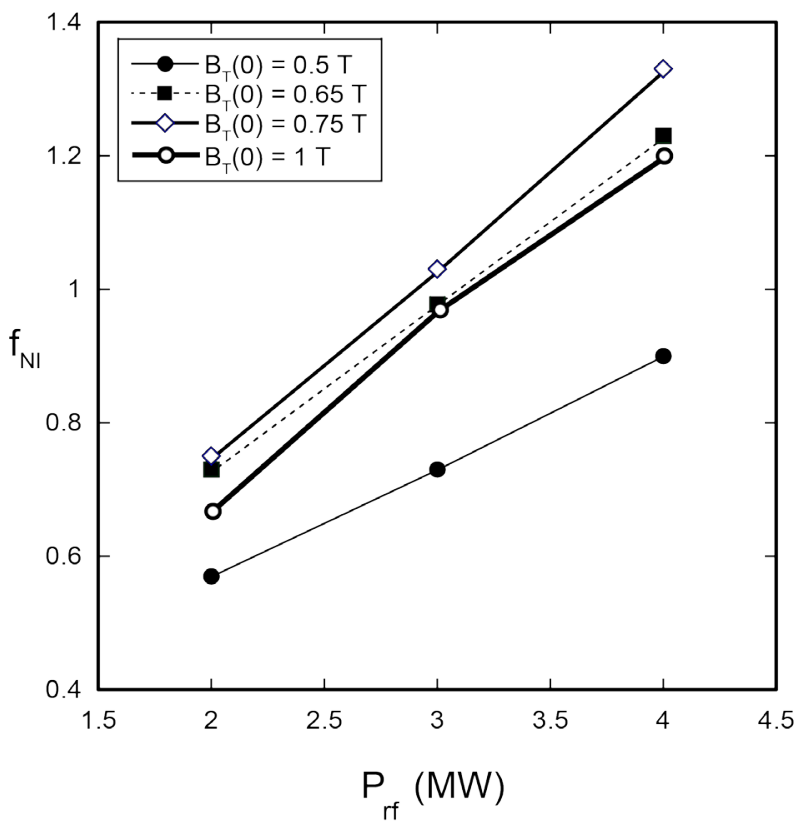

Fig. 7. $f_{N I}$ versus $P_{r f}$ for the plasma simulations with $n_{e}(0)=1.43 \times 10^{19} \mathrm{~m}^{-3}$.

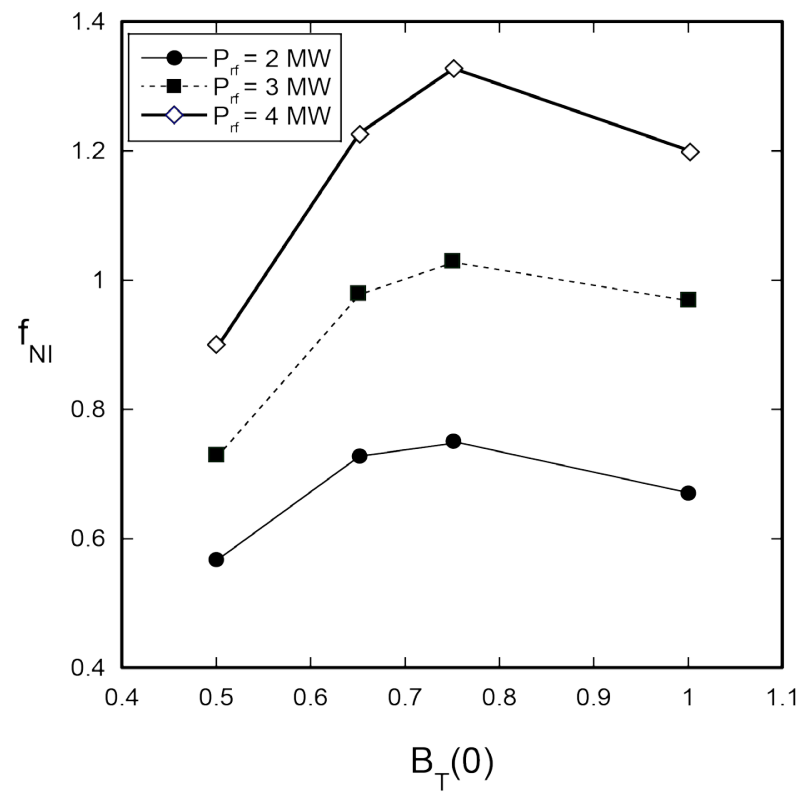

Fig. 8. $f_{N I}$ versus $B_{T}(0)$ for the plasma simulations with $n_{e}(0)=1.43 \times 10^{19} \mathrm{~m}^{-3}$.

As mentioned earlier, increasing $n_{e}(0)$ to $1.43 \times 10^{19} \mathrm{~m}^{-3}$ provided stable plasma simulations up to $P_{r f}=4 \mathrm{MW}$ at $B_{T}(0)=1 \mathrm{~T}$. Figure 7 plots $f_{N I}$ at $0.4 \mathrm{~s}$ versus $P_{r f}$ for this slightly higher density case. $\mathrm{f}_{\mathrm{NI}} \geq 1$ is predicted for $P_{r f} \geq 3 \mathrm{MW}$ for the plasma simulations with $B_{T}(0)=0.75 \mathrm{~T}$. At $P_{r f}=4 \mathrm{MW}$ all the plasma simulations with $B_{T}(0) \geq 0.65$ predict fully non-inductive operation. Increasing $P_{r f}$ from 2 to $4 \mathrm{MW}$ increased $f_{N I}$ by $1.6,1.7$, 1.8 and 1.4 for the $0.5 \mathrm{~T}, 0.65 \mathrm{~T}, 0.75 \mathrm{~T}$ and $1 \mathrm{~T}$ simulations, respectively. As expected, the somewhat higher density reduced the $f_{N I}$ achieved at a given $P_{r f}$ and $B_{T}(0)$ compared to the lower density simulations.

Figure 8 plots $f_{N I}$ at $0.4 \mathrm{~s}$ versus $B_{T}(0)$ for the $n_{e}(0)=1.43 \times 10^{19} \mathrm{~m}^{-3}$ simulations. While $f_{N I}$ increases significantly when $B_{T}(0)$ is raised from $0.5 \mathrm{~T}$ to $0.65 \mathrm{~T}$, $\mathrm{f}_{\mathrm{NI}}$ decreases when $B_{T}(0)$ increases from 0.75 to $1 \mathrm{~T}$, as predicted for the $n_{e}(0)=1.15 \times 10^{19} \mathrm{~m}^{-3}$ simulations.

\section{Conclusions}

The TRANSP simulation results presented here strongly support the possibility of achieving stable $f_{N I} \geq 1$ plasmas with $P_{r f}=2-3 \mathrm{MW}$ in an $I_{p}=300 \mathrm{kA}$ NSTX-U plasma. However while increasing $P_{r f}$ to higher levels than were achieved on NSTX shot \#138506 is predicted to increase $f_{N I}$ the simulations also predict that the plasma may become unstable as $P_{r f}$ is increased, particularly at the highest $B_{T}(0)$ values achievable on NSTX-U. The simulations predict that increasing $B_{T}(0)$ to $0.65 \mathrm{~T}$ from $0.55 \mathrm{~T}$, the value used in NSTX experiment, may significantly increase the achievable $f_{N I}$. One caveat is that operationally it was difficult to control $I_{p}=300 \mathrm{kA}$ NSTX plasmas when fast wave power was coupled into the $I_{p}$ flattop, even at $P_{r f}=1.4 \mathrm{MW}$. Coupling fast wave power at even higher levels will no doubt be challenging. But even more challenging will be coupling fast wave power immediately after start up in order to ramp $I_{p}$ up to 300-400 kA. These TRANSP simulation predictions must now await experimental validation in NSTX-U.

This work was supported by United States Department of Energy contract no. DE-AC02-09CH11466.

\section{References}

1. Y.-K.M. Peng, et al., Fusion Sci. Technol. 56, 957 (2009)

2. J. E. Menard, et al., Nucl. Fusion 52, 083015 (2012)

3. F. M. Poli, et al., Nucl. Fusion 55, 123011 (2015)

4. G. Taylor, et al., Phys. Plasmas 19, 042501 (2012)

5. R. Andrei, Bull. Am. Phys. Soc. 57, 12 (2012)

6. M. Brambilla, Plasma Phys. Control. Fusion 44, 2423 (2002)

7. T. Rafiq, et al., Phys. Plasmas 20, 032506 (2013)

8. S. P. Gerhardt, et al., Nucl. Fusion 51, 073031 (2011) 